\title{
Psicobiología de la Creatividad, Emociones y Actitud Creativa
}

\section{Psychobiology of Creativity, Emotions and Creative Attitude}

\author{
Julio C. Penagos-Corzo * \\ Universidad de las Américas Puebla, México
}

\begin{abstract}
La creatividad como fenómeno psicológico implica procesos moleculares, en el sentido estricto del término, así como procesos globales que pueden contemplar interacción social o cultural. El estudio de la creatividad, desde las neurociencias, ha arrojado hallazgos que van desde lo genético hasta lo neuroanatómico. Sin embargo, es probable que tales hallazgos tengan una comprensión incompleta, o incluso errónea, si no se consideran la mediación de otras variables. El objetivo del presente trabajo es vincular el procesamiento emocional y la actitud creativa con las variables psicobiológicas asociadas a la creatividad. Para ello, se hace una revisión de la literatura en la que se destacan algunas aproximaciones a la creatividad desde las neurociencias. Se consideró que fueran trabajos meta-analíticos o de revisión crítica, así como trabajos empíricos, que en ambos casos estuvieran indizados en Web of Science y que tuvieran al menos dos citas por año, salvo los estudios de 2017. Como resultado del análisis de estudios genéticos, de neurotransmisión y otros a nivel de neuroimagen y electroencefalografía se señalan posibles vínculos con hallazgos de trabajos sobre procesamiento emocional. La actitud creativa a su vez, al orientar los desempeños creativos, da un sentido más comprehensivo a tal vinculación. Finalmente se discuten los hallazgos de la neurociencia en términos la necesidad de brindar marcos de referencia más acotados que permitan mejores explicaciones.
\end{abstract}

Descriptores: Creatividad; Actitud; Afectividad; Biología; Psicología.

Creativity as a psychological phenomenon implies molecular processes, in the strict sense of the term, as well as global processes that can contemplate social or cultural interaction. The study of creativity, from the neurosciences perspective, has yielded findings that range from the genetic to the neuroanatomical. However, such findings are likely to have incomplete, or even erroneous, understanding if mediation of other variables is not considered. The objective of this work is to link emotional processing and creative attitude with the psychobiological variables associated with creativity. To do this, a review of the literature is made in which some approaches to creativity from the neurosciences are highlighted. It was considered that they were metaanalytic or critical review works, as well as empirical studies, which in both cases were indexed in Web of Science and had at least two citations per year, except for studies of 2017. As a result of the analysis of genetic studies, neurotransmission and others at the level of neuroimaging and electroencephalography, possible links to findings of work on emotional processing are indicated. The creative attitude, in turn, guiding the creative performances, gives a more comprehensive sense to such linkage. Finally, the findings of neuroscience are discussed in terms of the need to provide more limited frameworks that allow better explanations.

Keywords: Creativity; Attitudes; Emotions; Biology; Psychology.

*Contacto: julioc.penagos@udlap.mx

ISSN: 2254-3139

www.rinace.net/riejs/

revistas.uam.es/riejs
Recibido: $\quad 3$ de septiembre 2017

$1^{\text {a }}$ Evaluación: 15 de diciembre 2017

$2^{\text {a }}$ Evaluación: 7 de enero 2018

Aceptado: 16 de abril 2018 


\section{Introducción}

La creatividad como fenómeno psicológico implica procesos moleculares, en el sentido estricto del término, así como procesos globales que pueden contemplar interacción social o cultural. Todos los procesos, desde una perspectiva psicológica, terminan en conducta. La conducta creativa se convierte entonces en el producto de las fuerzas que permiten a la creatividad. La conducta creativa es lo que generalmente se ve o se mide. Incluso cuando se habla de pensamiento. El pensamiento creativo es medido en desempeño, es decir en conducta. Así la flexibilidad cognoscitiva (indicador frecuente de creatividad) se transforma, por ejemplo, en la conducta de identificar categorías, permanecer o cambiar entre ellas. El abordaje debe considerarse con sumo cuidado pues es probable que generalizaciones en torno a la creatividad provengan de un indicador, a veces más relevante, a veces menos.

Es frecuente que la creatividad esté definida en términos de lo original y lo útil o relevante (Gajda, Karwowski y Beghetto, 2017; Runco y Jaeger, 2012). Como constructo psicológico su abordaje puede ser diverso. En este sentido, a partir del trabajo de Rhodes (1961), se ha tratado de agrupar tal diversidad en tres aproximaciones teóricas: proceso, persona, producto. Sin embargo, a la fecha tal clasificación resulta acotada pues el nivel de profundidad y análisis en el estudio de la creatividad ha crecido. Por ello, la propuesta de Hennessey y Amabile (2010) probablemente resulta más útil. Estas autoras realizan una aproximación en donde los enfoques en el estudio de la creatividad están ordenados en una especie de círculos concéntricos que relativamente identifica niveles de análisis. Tales niveles van desde lo neurológico (el círculo más interno), hasta las aproximaciones sintéticas (el círculo más externo). Entre estos, en orden ascendente están: entrenamiento/afecto/cognición, personalidad/individuo, grupos, ambiente social y sociedad/cultura. Hennessey y Amabile (2010) claramente indican que los niveles son una forma de simplificación pues éstos se cruzan y la totalidad del proceso creativo es más que la suma de sus partes. Sin embargo, el trabajo antes citado ayuda a dimensionar la multiplicidad de aproximaciones y a ilustrar la complejidad del fenómeno creativo. Pues hace evidente que cualquier aproximación sólo logrará, en el mejor de los casos, clarificar una mínima parte de algún tema específico de uno de los niveles propuestos. Si bien los niveles se entrecruzan, el nivel en el que el investigador de la creatividad trabaje, probablemente sea el que se considere más relevante. En el presente trabajo se aborda básicamente el primer nivel (neurológico) (cuadro 1), pero se trata de esclarecer cómo aspectos de otro nivel (afectivo/cognitivo) no sólo sirven de eslabones sino probablemente contribuyen a la explicación de los niveles con los que más claramente se enlazan.

Para realizar este abordaje se hace, en el presente trabajo, una revisión de la literatura; como criterio relevante para la inclusión de estudios neurocientíficos se consideró que fueran trabajos meta-analíticos o de revisión crítica, así como trabajos empíricos, que en ambos casos estuvieran indizados en Web of Science y que tuvieran al menos dos citas por año, salvo los estudios de 2017. Es importante destacar que en promedio los trabajos analizados cuentan con alrededor de 20 citaciones en revistas con factor de impacto. Estos criterios, pueden ser considerados como indicadores de notoriedad o relevancia académica y/o científica. 


\section{Indicadores de creatividad en neurociencias}

Cuando la creatividad es estudiada desde las neurociencias, en general quedará definida por los procedimientos que se usen para medirla. Sean estos procedimientos tareas o tests. Los investigadores, son cautelosos y es frecuente que señalen que lo que alcanzaron a medir es un indicador de creatividad y no la creatividad misma. Así, con esta óptica se pueden encontrar estudios que tratan de abordar a la creatividad, sin embargo, la medición es de una variable relacionada; por ejemplo, el pensamiento divergente o la flexibilidad (Swartwood, Swartwood y Farrell, 2003). Si bien es bastante aceptado que la flexibilidad es un indicador de creatividad (Runco, 2004), la definición del indicador en mención puede plantear un importante problema. No es lo mismo la aproximación de Guilford en torno a la flexibilidad (Almeida et al., 2008), que hacer un cambio de tareas (task switching o setshifting). En este enfoque, la flexibilidad se refiere a una función ejecutiva en donde la atención se cambia de una tarea a otra (Barcelo et al., 2006).

En diversas aproximaciones a la creatividad desde las neurociencias, la forma de atención antes mencionada se ha medido por medio del Wisconsin Card Sorting Test (WCST). Dicho instrumento se ha usado para múltiples propósitos, y de manera frecuente para lesiones en lóbulos frontales (Demakis, 2003). Sin embargo, existen cuestionamientos sobre la validez y confiabilidad de dicho instrumento (Barceló y Santomé-Calleja, 2000). Además, su reducida validez ecológica (Chiu et al., 2017) probablemente contribuye a las diferencias encontradas entre diferentes poblaciones (Kohli y Kaur, 2006). Con esta información, resultan por tanto bienvenidas otras formas de medida.

Las tareas de pensamiento divergente son frecuentemente usadas para relacionar variables neurobiológicas con la creatividad. Por ejemplo, se ha reportado que personas bajo entrenamiento en pensamiento divergente cuando son medidas con una tarea de nombrar usos inusuales de un objeto, en comparación con usos ordinarios, muestran mayor activación en las circunvoluciones supramarginal, angular, y media temporal (Kleibeuker et al., 2017). En otro estudio, también de estimulación cognitiva, pero en este caso vía la exposición a ideas de otros, se encontró que dicha estimulación tiene efectos positivos en algunos indicadores de la creatividad. Se observaron aparejados a estos efectos el involucramiento de áreas cerebrales relacionadas con los procesos de atención, integración semántica y memoria (Fink et al., 2010). Además de las tareas de usos alternativos, otros procedimientos similares se han usado, tales como invención de nombres. En este caso se les dan a los participantes letras iniciales de personajes ficticios y ellos tienen que inventar nombres tan originales como puedan con estas iniciales. Con estas tareas en comparación con tareas de control se ha observado una sincronización alfa en regiones corticales parietales, así como una alta activación en las regiones frontales del hemisferio izquierdo (Fink et al., 2009). Aun así, la generalización resulta compleja. Es probable que inventar nombres tenga alguna relación con la creatividad en la vida cotidiana, pero en el mejor de los casos es sólo un indicador.

Lo anterior puede ayudar a ejemplificar que cuando se habla de indicadores de creatividad quizá el camino para confirmar tales indicadores es todavía largo. Aunque el problema metodológico que plantea la tarea o el indicador pareciera estar acotado por el nivel de validez de estos, el problema tiene un carácter más sustantivo ¿cuál es el estado afectivo al momento de hacer la tarea? ¿este estado afectivo es diferencial entre un momento creativo de uno que no lo es? Es posible plantear una sencilla aproximación de repuesta: sí lo es. Una respuesta lúdica, de resolución de problemas, o de éxito, se convierte en una 
respuesta de logro. Estas respuestas tienen correlatos fisiológicos claros. Uno de ellos es la activación de los circuitos de recompensa, en donde la modulación de la dopamina es de particular importancia (Skvortsova et al., 2017; Vrieze et al., 2013).

\subsection{Genes y neurotransmisores asociados a la creatividad}

Dentro de lo que se podría llamar estudio molecular de los procesos creativos está la búsqueda de relación entre creatividad y niveles de dopamina (Boot et al., 2017; Flaherty, 2005) o la posible participación del gen receptor dopaminérgico D2 y del TPHI gen (Reuter et al., 2006). Igualmente se ha explorado la participación del gen que codifica la neuregulina en personas con altos niveles de creatividad (Kéri, 2009). El gen también se ha vinculado con la esquizofrenia, en donde una posible explicación está en la alteración del sistema dopaminérgico (Neddens et al., 2009). Si bien las evidencias son todavía escasas, se han encontrado relaciones entre tareas de pensamiento divergente, asociación remota y frecuencia de parpadeo (eye blink rate), que es un marcador clínico de funcionamiento dopaminérgico (Chermahini y Hommel, 2010). Sin embargo, estudios bien controlados sugieren que los resultados deben considerarse con cautela. Por ejemplo, Runco et al. (2011) al investigar las posibles relaciones entre diversos genes y creatividad, encontraron que la relación aparente entre algunos genes (COMT, DAT, DRD4, y TPH1) y creatividad, en realidad tal relación es únicamente con la fluidez ideacional, que no es el mejor indicador de creatividad. Aun así, los resultados son contradictorios, pues en otro estudio se encontraron relaciones entre los genes TPH1, ТPH2 e indicadores de creatividad como flexibilidad y originalidad, además de fluidez (Zhang y Zhang, 2007). Lo anterior además abre la posibilidad al papel de la serotonina en la creatividad.

\subsection{El papel de las emociones}

Como se señaló arriba, la relación entre dopamina y creatividad se ha explorado; no obstante, parece ser menor la exploración entre serotonina y creatividad. Los genes TPH 1 y TPH2 son genes serotoninérgicos. Es probable que la relación entre dopamina y creatividad contribuya a la capacidad asociativa. Sin embargo, si la serotonina está implicada (Volf et al., 2009), posiblemente su participación media a la creatividad más en un sentido emocional que en un sentido estrictamente cognoscitivo. De hecho, se ha hipotetizado que la serotonina tiene un papel de integración o modificación de la información emocional (Cools, Roberts y Robbins, 2008) que conduce a un monitoreo conductual que termina por afectar el proceso de la toma de decisiones (Homberg, 2012).

Las implicaciones de esto son relevantes, pues las conclusiones sobre el nivel neurológico explorado de la creatividad quizá no están considerando que el hallazgo tiene una variable concomitante: un estado afectivo que además tiene una psicofisiología asociada. Así, los hallazgos que vinculan a ciertos neurotransmisores con creatividad, quizá sean muestra de indicadores moleculares de otras variables que están presentes en el momento creativo, por ejemplo, un estado de ánimo positivo.

En este sentido existe evidencia que señala una correlación positiva entre niveles de serotonina y estado de ánimo positivo (Young, 2007), así como también hay relación entre afecto positivo y desempeño creativo (Amabile et al., 2005). Algo similar puede observarse en relación con la dopamina. Por ejemplo, Chermahini y Hommel (2012) reportan que es común que se asuma que el estado de ánimo positivo mejora la creatividad y que la dopamina interviene en esta relación. Los datos recolectados por los autores citados se inclinan a esta probable mediación. En su estudio, el índice de parpadeo ocular fue medido 
como indicador de dopamina y observan que este indicador tiene un correlato tanto con las emociones como con la creatividad, pero apuntan a que no es el estado de ánimo lo que favorece la creatividad sino la dopamina. Estas conclusiones pueden ser apoyadas por evidencias de tipo experimental en donde se observa que el bloqueo del receptor D2 de la dopamina tiene efectos en el estado de ánimo, en la flexibilidad, así como en la asimetría alfa electroencefalográfica (Wacker, 2017). Este último punto merece una mención aparte.

\subsection{Neuroimagen y EEG}

Las aproximaciones moleculares en torno a la creatividad son más bien recientes. En cambio, las aproximaciones basadas en EEG o incluso neuroimagen tienen más años de desarrollo y hay abundante información (Penagos-Corzo, 2017). Por sus propias características, tanto los estudios con EEG o neuroimagen han estado más enfocados a los aspectos cognoscitivos de la creatividad que al procesamiento emocional.

En relación con la neuroimagen, es frecuente encontrar estudios enfocados en localizar las regiones encefálicas vinculadas al proceso creativo. Las evidencias en torno a la participación de ciertas regiones son diversas. Sin embargo, se ha sugerido a la corteza prefrontal ventromedial (CPFVM) como el sitio de origen de la creatividad (Chakravarty, 2012), aunque también se ha señalado que tal afirmación puede ser una sobregeneralización debido, entre otras razones, a la falta de evidencias (Prakash y Zhongde, 2013); además, existe abundante evidencia sobre otra región cercana: la corteza prefrontal dorso lateral (CPFDL) (Aziz-Zadeh, Liew y Dandekar, 2013; Gilbert et al., 2010; Liu et al., 2015). Sobre esta región un reciente estudio ha logrado hacer una demostración experimental con estimulación transcraneal. Los autores de dicho estudio encontraron que, en la condición de supresión de la CPFDL, los participantes mejoraron su desempeño en la solución creativa a un problema, en comparación con las condiciones de estimulación o de no estimulación (Luft et al., 2017).

Tanto la CPFVM como la CPFDL tienen un papel relevante en la regulación emocional (Fan et al., 2013); por ejemplo, se ha reportado que al aumentar su actividad existe mayor autocontrol (Hare, Camerer y Rangel, 2009). Lo que resulta congruente con los hallazgos de Luft y colaboradores (2017), pues al suprimir a la CPFDL probablemente hubo menos control inhibitorio que facilitó pensar "fuera de la caja".

Por otro lado, estudios con EEG, han reportado correlatos entre poder alfa y creatividad (Pidgeon et al., 2016). De hecho, se ha afirmado que la correlación positiva entre el poder de la banda alfa y la creatividad es uno de los hallazgos más robustos y consistentes en el estudio de los procesos creativos desde las neurociencias (Fink y Benedek, 2014). Aquí también destaca la probable mediación emocional en torno a las variables en mención. Por ejemplo, se sabe que el poder alfa en zonas frontales está vinculado a la reactividad emocional (Benca et al., 1999). Además, en un trabajo reciente se indica que en cinco emociones positivas (amor, gratitud, esperanza, inspiración y orgullo) llamadas de "ánimo" o "aliento" por los autores del estudio, se observaron fuertes correlaciones positivas en el poder de las banda alfa y beta (Hu et al., 2017). Al menos una de ellas, la inspiración, resulta claramente relevante en el proceso creativo. 
Cuadro 1. Trabajos empíricos representativos de los estudios neurocientíficos

\begin{tabular}{|c|c|c|c|}
\hline REFERENCIA & MÉTODo & $\begin{array}{c}\text { MEDIDAS DE } \\
\text { CREATIVIDAD } \\
\end{array}$ & HALLAZGOS MÁS RELEVANTES \\
\hline $\begin{array}{l}\text { Reuter et al. } \\
(2006)\end{array}$ & $\begin{array}{l}\text { Análisis genético } \\
\text { (DNA extraído de } \\
\text { células bucales). }\end{array}$ & $\begin{array}{l}\text { Subtest } \\
\text { Inventiveness del } \\
\text { Berlin Intelligence } \\
\text { Structure Test. }\end{array}$ & $\begin{array}{l}\text { Genes DRD2 y TPH están asociados a la } \\
\text { creatividad, mientras que COMT no } \\
\text { mostró relación alguna. }\end{array}$ \\
\hline Kéri (2009) & $\begin{array}{l}\text { Análisis genético } \\
\text { (DNA fue extraído de } \\
\text { una muestra de } \\
\text { sangre) }\end{array}$ & $\begin{array}{l}\text { Test Creative } \\
\text { Achievement, y } \\
\text { el subtest Just } \\
\text { Supose del } \\
\text { Torrance Test of } \\
\text { Creative Test. }\end{array}$ & $\begin{array}{l}\text { Genotipos promotores de la } \\
\text { neuregulina } 1 \text {, revelaron efectos } \\
\text { significativos para cada medida de } \\
\text { creatividad }\end{array}$ \\
\hline $\begin{array}{l}\text { Chermahini } \\
\text { y Hommel } \\
(2010)\end{array}$ & $\begin{array}{l}\text { Marcador biológico: } \\
\text { Frecuencia de } \\
\text { parpadeo (EBR por } \\
\text { sus siglas en inglés) }\end{array}$ & $\begin{array}{l}\text { Test de usos } \\
\text { alternativos de } \\
\text { Guilford }\end{array}$ & $\begin{array}{l}\text { Se encontró una relación en forma de } \\
\text { U invertida entre EBR y flexibilidad. }\end{array}$ \\
\hline $\begin{array}{l}\text { Runco et al. } \\
(2011)\end{array}$ & $\begin{array}{l}\text { Análisis genético } \\
\text { (DNA extraído de } \\
\text { células bucales). }\end{array}$ & $\begin{array}{l}\text { Test de usos } \\
\text { alternativos de } \\
\text { Guilford. }\end{array}$ & $\begin{array}{l}\text { Los genes DAT, COMT, DRD4, DRD2 y } \\
\text { TPH } 1 \text { tienen un papel predictor para la } \\
\text { fluidez. DAT tuvo efectos en } \\
\text { flexibilidad. }\end{array}$ \\
\hline $\begin{array}{l}\text { Zhang y } \\
\text { Zhang } \\
(2007)\end{array}$ & $\begin{array}{l}\text { Análisis genético } \\
\text { (DNA extraído de una } \\
\text { muestra de sangre). }\end{array}$ & $\begin{array}{l}\text { Test de usos } \\
\text { alternativos de } \\
\text { Guilford. }\end{array}$ & $\begin{array}{l}\text { TPH } 1 \text { y TPH2 están relacionados } \\
\text { significativamente, con todas las } \\
\text { medidas del test. }\end{array}$ \\
\hline $\begin{array}{l}\text { Aziz-Zadeh } \\
\text { Liew y } \\
\text { Dandekar } \\
(2013)\end{array}$ & $\begin{array}{l}\text { FMRI, condición } \\
\text { creativa vs condición } \\
\text { control vs condición } \\
\text { descanso. }\end{array}$ & $\begin{array}{l}\text { Tarea de } \\
\text { pensamiento } \\
\text { divergente para } \\
\text { manipulación } \\
\text { mental de } \\
\text { formas. }\end{array}$ & $\begin{array}{l}\text { La tarea creativa activa } \\
\text { significativamente a la corteza parietal } \\
\text { posterior, la corteza premotora, la } \\
\text { corteza prefrontal dorsolateral } \\
\text { (DLPFC) y la corteza prefrontral medial } \\
\text { (PFC) del hemisferio izquierdo. }\end{array}$ \\
\hline $\begin{array}{l}\text { Gilbert et al. } \\
(2010)\end{array}$ & $\begin{array}{l}\text { FMRI, comparación } \\
\text { de tareas poco } \\
\text { estructuradas. }\end{array}$ & $\begin{array}{l}\text { Tareas de diseño } \\
\text { y solución de } \\
\text { problemas. }\end{array}$ & $\begin{array}{l}\text { DLPFC tiene un papel importante en } \\
\text { tareas poco estructuradas, y muestra } \\
\text { mayor actividad en tareas de diseño } \\
\text { que en solución de problemas. }\end{array}$ \\
\hline $\begin{array}{l}\text { Liu et al. } \\
(2015)\end{array}$ & $\begin{array}{l}\text { FMRI. Comparación } \\
\text { de dos fases del } \\
\text { proceso creativo } \\
\text { entre expertos y } \\
\text { novatos. }\end{array}$ & $\begin{array}{l}\text { Composición } \\
\text { poética. }\end{array}$ & $\begin{array}{l}\text { La corteza medial prefrontal (MPFC) } \\
\text { mostró mayor actividad en dos fases } \\
\text { relevantes del proceso creativo no así } \\
\text { la DLPFC. Los expertos mostraron una } \\
\text { desactivación significativamente más } \\
\text { fuerte en DLPFC, aunque con patrones } \\
\text { generales similares en ambos grupos. }\end{array}$ \\
\hline $\begin{array}{l}\text { Luft et al. } \\
(2017)\end{array}$ & $\begin{array}{l}\text { Estimulación } \\
\text { transcraneal de } \\
\text { corriente directa } \\
\text { (TDCS) (supresión de } \\
\text { la DLPFC). }\end{array}$ & $\begin{array}{l}\text { Problemas } \\
\text { aritméticos. }\end{array}$ & $\begin{array}{l}\text { La TDCS sobre la DLPFC izquierda } \\
\text { facilita la solución de problemas } \\
\text { nuevos, al relajar las normas } \\
\text { aprendidas. }\end{array}$ \\
\hline $\begin{array}{l}\text { Fink et al. } \\
(2017)\end{array}$ & $\begin{array}{l}\text { Electroencefalografía } \\
\text { durante ideación } \\
\text { creativa } \\
\text { convencional y } \\
\text { reevaluación } \\
\text { cognoscitiva. }\end{array}$ & $\begin{array}{l}\text { Test de usos } \\
\text { alternativos. } \\
\text { Reappraisal } \\
\text { Inventiveness } \\
\text { Test. }\end{array}$ & $\begin{array}{l}\text { Tanto la reevaluación cognitiva como } \\
\text { la ideación creativa verbal } \\
\text { convencional tienen un patrón de } \\
\text { poder alfa similar. Sin embargo, la } \\
\text { primera tiene incremento } \\
\text { significativamente de dicho poder en } \\
\text { sitios prefrontales, junto con } \\
\text { incrementos alfa menores en los sitios } \\
\text { corticales posteriores. }\end{array}$ \\
\hline
\end{tabular}

Fuente: Elaboración propia. 
Así, es posible denotar, aún más, que los hallazgos sobre creatividad, quizá también están reportando, sin proponérselo, hallazgos vinculados indirectamente al procesamiento emocional en el momento creativo. Fink y colaboradores (2017) abordan directamente esta posibilidad. Para ello, miden el desempeño creativo en un contexto de regulación emocional similar a la vida diaria. Los hallazgos confirman que el poder alfa tiene correlaciones positivas con la creatividad a la vez que incrementos en dicho poder implican efectos en el estado emocional.

\section{Actitud creativa}

Líneas arriba se citó un trabajo sobre algunas emociones positivas denominadas de "aliento" o de "ánimo" por sus autores (Hu et al., 2017). Tal vez no sea arriesgado ubicarlas a la vez como actitudes. Desde algunos enfoques las actitudes pueden tener componentes cognoscitivos, conductuales y emocionales, además de ser una inclinación a responder de alguna manera (Myers y Twenge, 2012). Así, por ejemplo, la gratitud, citada en el trabajo como una emoción, también aparece como una actitud en otro lugar (Diesen, 2016). Lo mismo puede decirse de la esperanza (Reynolds, 2008) o incluso del orgullo (Haut, Prohl y Emrich, 2016). El señalamiento en torno a las emociones como actitud es relevante, debido a que, si bien el procesamiento emocional parece ser una variable mediadora en el proceso creativo, la actitud creativa resulta tan significativa que se ha sugerido que puede ser el origen de la creatividad misma (Penagos-Corzo, 2014). Es probable que no se pueda demostrar a la fecha un origen de la creatividad (si es que la hay), pero sí identificar variables que juegan un papel de alta relevancia; en este caso, la actitud.

En el acto de crear, las propias creencias sobre la creatividad no parecen ser determinantes para ésta; pues a pesar de que las persona tienen fuertes creencias de cómo facilitar su creatividad, éstas no resultan congruentes con lo que se observa en su experiencia (Baas et al., 2015). Lo anterior refleja lo que se pudiera llamar actitud hacia la creatividad. Tal actitud "hacia" es diferente de la actitud creativa. En ésta, la orientación está imbuida. Lo creativo es un adjetivo o incluso, en su conjunto, un sustantivo que tiene un objeto, por ejemplo, la resolución de un problema. Así, alguien puede tener una actitud creativa cuando se enfrenta a un evento sin respuesta y se ensayan múltiples respuestas para ese evento. Por ello se afirma que la actitud creativa es un conglomerado de evaluaciones, afectos y comportamientos proactivos, primordialmente flexibles, tenaces en la incertidumbre, orientados a la generación de ideas o acciones, que resultan socialmente relevantes (Penagos-Corzo, 2014). En la definición parece faltar un elemento esencial: la originalidad. Ésta será un producto. En la actitud en mención no está la originalidad per se, pues es, quizá, una propiedad emergente.

Asumiendo que la actitud orienta los afectos, las cogniciones o las conductas en el acto de crear, la orientación afectiva, de acuerdo al autor de la última cita, incluye el maravillarse o sorprenderse (wondering) y a la motivación, principalmente la motivación intrínseca (Penagos-Corzo, Olvera y Pintado, 2017). Sin embargo, quizá este afecto deba incluir, además de la valencia emocional positiva o negativa, emociones más complejas. Incluso se ha encontrado que los tipos de amor propuestos por Sternberg (1986), tienen correlatos con la creatividad (Campbell y Kaufman, 2017); tanto correlaciones negativas (creatividad artística), como correlaciones positivas (creatividad en la vida diaria). Si bien se ha sugerido que estados afectivos negativos como la depresión pueden tener efectos beneficiosos en la creatividad (Heilman, Nadeau y Beversdorf, 2003), la evidencia meta- 
analítica indica que los estados afectivos positivos son más favorables (Baas et al., 2008; Johnson et al, 2012).

Evidencias experimentales apuntan a que el estado de ánimo positivo abarca otras esferas de la cognición como un incremento en las capacidades de asociación y atención, probablemente asociados a una reducción en el control inhibitorio durante la inducción emocional positiva (Rowe, Hirsh y Anderson, 2007). En este sentido, se ha reportado que un alto control inhibitorio puede tener efectos negativos en la solución de problemas (Cassotti et al., 2016). Así, no parece ser sólo el estado afectivo lo que favorece la solución de problemas, sino variables que están presentes en el estado afectivo. De hecho, existe evidencia que señala que más que el afecto por sí mismo, es la información transmitida por el estado afectivo lo que modifica la respuesta (Clore y Huntsinger, 2007). Esto puede ser congruente la propuesta de la actitud creativa, que incluye un componente cognoscitivo (Penagos-Corzo, 2014). Tal componente hace referencia a una cognición flexible, abierta y probablemente sobre-inclusiva. La sobre-inclusión cognoscitiva o el pensamiento sobreinclusivo sucede cuando una persona ubica en una misma categoría elementos que no forman parte de ella (Runco, 2007).

El maravillarse o sorprenderse, aunque vistos anteriormente como afectivos, muestra claramente la intersección con lo cognitivo. Por un lado, los procesos emocionales y cognoscitivos son prácticamente indisolubles (Damasio, 1994), y, por otro lado, la persona cuando se "maravilla", ve, observa, de forma particular la situación o evento que es objeto de su actitud creativa. En este sentido, se ha postulado que la observación es un mecanismo especial en el proceso creativo que permite relacionar eventos que parecen no estar vinculados y se ven de forma diferente (Gaymer, 1985). Evidencias recientes confirman la última afirmación: en una serie de experimentos un grupo de participantes fue expuesto a diferentes tareas de percepción binocular (Antinori, Carter y Smillie, 2017). Los resultados del estudio antes citado indican que las personas con mayor apertura (un predictor de creatividad) tienen mejores desempeños en las tareas visuales de rivalidad binocular, a la vez que sugieren que las tendencias creativas se extienden hasta los procesos visuales básicos. Más aún, también se encuentra una correlación positiva entre el estado de ánimo positivo y desempeño visual, principalmente en las personas con mayor apertura. Ver algo implica un enfoque de la atención y al hacerlo quien ve elige, no siempre conscientemente, lo que ve. Un ejemplo de esto es la ceguera inatencional. Ésta se produce cuando las personas no se dan cuenta de un estímulo inesperado, pero saliente, que aparece en su entorno inmediato mientras participan en una tarea (O'Shea y Fieo, 2015). Ver implica elegir qué ver y al parecer también cómo ver. Ese "cómo" ver, en la actitud creativa es un ver flexible y abierto.

\section{Conclusiones}

En este trabajo se propuso dilucidar cómo las emociones pueden ser una variable subyacente en las aproximaciones neurobiológicas en el estudio de la creatividad y cómo la actitud creativa igualmente juega un papel relevante. En este abordaje, la flexibilidad y el pensamiento divergente resultan en indicadores relativamente frecuentes para medir la creatividad. Sin embargo, ambos presentan limitaciones en su validez. A nivel neurobiológico destacan los hallazgos tanto a nivel dopaminérgico como serotoninérgico. En ambos casos es posible establecer conexiones con procesos emocionales. Por otro lado, parece existir abundante evidencia a nivel de neuroimagen sobre diversas regiones, 
principalmente la CPFVM y la CPFDL. Tales regiones tienen un impacto en la regulación emocional; además, la supresión de actividad en la CPFDL mencionada anteriormente, sugiere menor control inhibitorio. Estudios con EEG también reportan basta evidencia, principalmente en relación al poder de la banda alfa. En este sentido, se ha sugerido que dicha banda está asociada a emociones positivas. Algunas de las emociones positivas mencionadas en este trabajo, pueden ser vinculadas, o incluso identificadas, como actitudes. Así, es posible señalar que tanto las emociones como las actitudes contribuyen a la explicación de las variaciones encontradas en las variables neurobiológicas asociadas a la creatividad.

Habrá que sumar a la discusión la necesidad de contextualizar e incluso acotar los hallazgos de la neurociencia. Por ello, un llamado a la mesura sobre el uso de lo "neuro" puede ser oportuno, pues

$$
\begin{aligned}
& \text { por muy vistosos que sean los resultados de la investigación con neuroimagen, no está } \\
& \text { bien confundir a la gente con injustificadas expectativas que no hacen sino ocultar las } \\
& \text { graves dificultades que comporta el estudio de la mente, sus logros y capacidades, o el } \\
& \text { estudio de los fenómenos sociales. (García-Albea, 2011, p. 580) }
\end{aligned}
$$

Por otro lado, la actitud creativa, en conjunto con las emociones, quizá estén matizando buena parte de los desempeños creativos observados en el estudio neurobiológico de la creatividad. Si las emociones median el proceso creativo, las actitudes lo orientan y probablemente lo originan. Las implicaciones de esto adquieren otra dimensión si se considera que la actitud creativa no sólo es sustantiva en la persona que realiza el proceso creativo, sino incluso en quien tiene como deber estimularlo en otras personas. Por ejemplo, se ha sugerido que la pertinencia de que los docentes tengan una actitud abierta para estimular el potencial creativo (Chacón, 2005; Klimenko, 2008). Esto es de particular importancia, pues probablemente los procesos de enseñanza pueden ser más justos si se cuida el desarrollo de la creatividad (Murillo y Hérnadez-Castilla, 2014), a la vez que serán más éticos si se tienden puentes transdisciplinarios en donde colaboren neurocientíficos, psicólogos, educadores y filósofos (Codina, 2014).

Por la complejidad de la actitud creativa, no parece probable que en el corto plazo se pueda vincular a las neurociencias. Además, a pesar de todo el fascinante avance en la psicobiología de la creatividad, considerar sólo lo biológico es sobre-simplificar (Poblano, 1991), a la vez de que es muy importante tener presente que la biología más bien es un marco de acotamiento de lo cognoscitivo y es la cultura la que lo transforma. Entre estos dos grandes niveles, las emociones y la actitud creativa, sirven de eslabones, túneles y puentes.

Otros trabajos han revisado la relación entre diversas variables neurobiológicas y creatividad (Boot et al., 2017; Camfield, 2005; Dietrich y Kanso, 2010; Heilman et al., 2003; Pigeon et al., 2016; Sprugnoli et al., 2017). Sin embargo, las emociones y las actitudes no se han integrado explícitamente en tales revisiones. Empero, sí se ha sugerido la pertinencia de su incorporación en el contexto neurobiológico de la creatividad (PenagosCorzo, 2017; Sawyer, 2011). Por ello se considera que el presente trabajo contribuye al conocimiento al iniciar una aproximación en dicho sentido. Para terminar, es oportuno señalar que la biología de la creatividad quizá la antecede, la actitud creativa orienta y sostiene a las acciones del proceso creativo; las emociones median este proceso y la cultura lo enriquece y al hacerlo se enriquece. 


\section{Referencias}

Almeida, L. S., Prieto, L., Ferrando, M., Oliveira, E. y Ferrándiz, C. (2008). Torrance test of creative thinking: The question of its construct validity. Thinking Skills \& Creativity, 3(1), 53-58. https://doi.org/10.1016/j.tsc.2008.03.003

Amabile, T. M., Barsade, S. G., Mueller, J. S. y Staw, B. M. (2005). Affect and creativity at work. Administrative Science Quarterly, 50(3), 367-403. https://doi.org/10.2189/asqu.2005.50.3.367

Antinori, A., Carter, O. L. y Smillie, L. D. (2017). Seeing it both ways: Openness to experience and binocular rivalry suppression. Journal of Research in Personality, 68, 15-22. https://doi.org/10.1016/j.jrp.2017.03.005

Aziz-Zadeh, L., Liew, S. y Dandekar, F. (2013). Exploring the neural correlates of visual creativity. Social Cognitive and Affective Neuroscience, 8(4), 475-480. https://doi.org/10.1093/scan/nsso2 1

Baas, M., Koch, S., Nijstad, B. A. y De Dreu, C. W. (2015). Conceiving creativity: The nature and consequences of laypeople's beliefs about the realization of creativity. Psychology of Aesthetics, Creativity \& The Arts, 9(3), 340-354. https://doi.org/10.1037/a0039420

Baas, M., De Dreu, C. W. y Nijstad, B. A. (2008). A meta-analysis of 25 years of mood-creativity research: Hedonic tone, activation, or regulatory focus? Psychological Bulletin, 134(6), 779806. https://doi.org/10.1037/a0012815

Barceló, F. y Santomé-Calleja, A. (2000). A critical review of the specificity of the Wisconsin card sorting test for the assessment of prefrontal function. Revista de Neurología, 30(9), 855-864.

Barcelo, F., Escera, C., Corral, M. J. y Periáñez, J. A. (2006). Task switching and novelty processing activate a common neural network for cognitive control. Journal of Cognitive Neuroscience, 18(10), 1734-1748. https://doi.org/10.1162/jocn.2006.18.10.1734

Benca, R. M., Obermeyer, W. H., Larson, C. L., Yun, B., Dolski, I., Kleist, K. D., ... y Davidson, R. J. (1999). EEG alpha power and alpha power asymmetry in sleep and wakefulness. Psychophysiology, 36(4), 430-436. https://doi.org/10.1111/1469-8986.3640430

Boot, N., Baas, M., Van Gaal, S., Cools, R. y De Dreu, C. W. (2017). Creative cognition and dopaminergic modulation of fronto-striatal networks: Integrative review and research agenda. Neuroscience and Biobehavioral Reviews, 78, 13-23. https://doi.org/10.1016/j.neubiorev.2017.04.007

Camfield, D. (2005). Neurology of exceptionality. En C. Stough (Ed.), Neurobiology of creativity (pp. 53-77). Nueva York, NY: Kluwer Academic.

Campbell, K. y Kaufman, J. (2017). Do you pursue your heart or your art? Creativity, personality, and love. Journal of Family Issues, 38(3), $287-311$. https://doi.org/10.1177/0192513X15570318

Cassotti, M., Agogué, M., Camarda, A., Houdé, O. y Borst, G. (2016). Inhibitory control as a core process of creative problem solving and idea generation from childhood to adulthood. New Directions for Child and Adolescent Development, 151, 61-72. https://doi.org/10.1002/cad.20153

Chacón, Y. (2005). Una revisión crítica del concepto de creatividad. Actualidades Investigativas en Educación, 5(1), 1-30.

Chakravarty, A. (2012). The neural circuitry of visual artistic production and appreciation: A proposition. Annals of Indian Academy of Neurology, 15(2), 71-75. https://doi.org/10.4103/0972-2327.94986 
Chermahini, S. A. y Hommel, B. (2010). The (b)link between creativity and dopamine: Spontaneous eye blink rates predict and dissociate divergent and convergent thinking. Cognition, 115(3), 458-465. https://doi.org/10.1016/j.cognition.2010.03.007

Chermahini, S. A. y Hommel, B. (2012). More creative through positive mood? Not everyone! Frontiers in Human Neuroscience, 6, 1-7. https://doi.org/10.3389/fnhum.2012.00319

Chiu, E., Wu, W., Hung, J. y Tseng, Y. (2017). Validity of the Wisconsin card sorting test in patients with stroke. Disability and Rehabilitation, 24(2), 1-5. https://doi.org/10.1080/09638288.2017.1378389

Clore, G. L. y Huntsinger, J. R. (2007). How emotions inform judgment and regulate thought. Trends in Cognitive Sciences, 11(9), 393-399. https://doi.org/10.1016/j.tics.2007.08.005

Codina, M. J. (2014). Neuroeducación: Reflexiones sobre neurociencia, filosofía y educación. Postconvencionales, 7(2), 164-181.

Cools, R., Roberts, A. C. y Robbins, T. W. (2008). Serotoninergic regulation of emotional and behavioural control processes. Trends in Cognitive Sciences, 12(1), 31-40. https://doi.org/10.1016/j.tics.2007.10.011

Damasio, A. R. (1994). Descartes' error: Emotion, reason, and the human brain. Nueva York, NY: Putnam's Sons.

Demakis, G. J. (2003). A meta-analytic review of the sensitivity of the Wisconsin card sorting test to frontal and lateralized frontal brain damage. Neuropsychology, 17(2), 255-264. https://doi.org/10.1037/0894-4105.17.2.255

Diesen, P. S. (2016). I feel lucky. Gratitude among young adults with phenylketonuria (PKU). Journal of Genetic Counseling, 25(5), 102-109. https://doi.org/10.1007/s 10897-015-9931-8

Dietrich, A. y Kanso, R. (2010). A review of EEG, ERP, and neuroimaging studies of creativity and insight. Psychological Bulletin, 136(5), 822-848. https://doi.org/10.1037/a0019749

Fan, F., Tan, S., Yang, F., Tan, Y., Zhao, Y., Chen, N., ... y Zuo, X. (2013). Ventral medial prefrontal functional connectivity and emotion regulation in chronic schizophrenia: A pilot study. Neuroscience Bulletin, 29(1), 59-74. https://doi.org/10.1007/s 12264-013-1300-8

Fink, A. y Benedek, M. (2014). EEG alpha power and creative ideation. Neuroscience and Biobehavioral Reviews, 44, 111-123. https://doi.org/10.1016/j.neubiorev.2012.12.002

Fink, A., Grabner, R. H., Benedek, M., Reishofer, G., Hauswirth, V., Fally, M., ... y Neubauer, A. C. (2009). The creative brain: Investigation of brain activity during creative problem solving by means of EEG and FMRI. Human Brain Mapping, 30(3), 734-748. https://doi.org/10.1002/hbm.20538

Fink, A., Grabner, R. H., Gebauer, D., Reishofer, G., Koschutnig, K. y Ebner, F. (2010). Enhancing creativity by means of cognitive stimulation: Evidence from an fMRI study. Neuroimage, 52(4), 1687-1695. https://doi.org/10.1016/j.neuroimage.2010.05.072

Fink, A., Weiss, E. M., Schwarzl, U., Weber, H., de Assunção, V. L., Rominger, C., ... y Papousek, I. (2017). Creative ways to well-being: Reappraisal inventiveness in the context of angerevoking situations. Cognitive, Affective \& Behavioral Neuroscience, 17(1), 94-105. https://doi.org/10.3758/s13415-016-0465-9

Flaherty, A. W. (2005). Frontotemporal and dopaminergic control of idea generation and creative drive. Journal of Comparative Neurology, 493(1), 147-153. https://doi.org/10.1002/cne.20768 
Gajda, A., Karwowski, M. y Beghetto, R. A. (2017). Creativity and academic achievement: A metaanalysis. Journal of Educational Psychology, 109(2), 269-299. https://doi.org/10.1037/eduo000133

García-Albea, J. E. (2011). Usos y abusos de lo 'neuro’. Revista de Neurología, 52(10), 577-580.

Gaymer, R. D. (1985), You are a camera... Some aspects of observation. The Journal of Creative Behavior, 19(1), 67-75. https://doi.org/10.1002/j.2162-6057.1985.tb00405.x

Gilbert, S. J., Zamenopoulos, T., Alexiou, K. y Johnson, J. H. (2010). Involvement of right dorsolateral prefrontal cortex in ill-structured design cognition: An fMRI study. Brain Research, 1312, 79-88. https://doi.org/10.1016/j.brainres.2009.11.045

Hare, T. A., Camerer, C. F. y Rangel, A. (2009). Self-control in decision-making involves modulation of the VMPFC valuation system. Science, 324(5927), 646-648. https://doi.org/10.1126/science.1168450

Haut, J., Prohl, R. y Emrich, E. (2016). Nothing but medals? Attitudes towards the importance of olympic success. International Review for the Sociology of Sport, 51(3), 332-348. https://doi.org/10.1177/1012690214526400

Heilman, K. M., Nadeau, S. E. y Beversdorf, D. O. (2003). Creative innovation: Possible brain mechanisms. Neurocase, 9(5), 369-379. https://doi.org/10.1076/neur.9.5.369.16553

Hennessey, B. A. y Amabile, T. M. (2010). Creativity. Annual Review of Psychology, 61(1), 569-598. https://doi.org/10.1146/annurev.psych.093008.100416

Homberg, J. R. (2012). Serotonin and decision making processes. Neuroscience and Biobehavioral Reviews, 36(1), 218-236. https://doi.org/10.1016/j.neubiorev.2011.06.001

Hu, X., Yu, J., Song, M., Yu, C., Wang, F., Sun, P., ... y Zhang, D. (2017). EEG correlates of ten positive emotions. Frontiers in Human Neuroscience, 11(26), 1-12. https://doi.org/10.3389/fnhum.2017.00026

Johnson, S. L., Murray, G., Fredrickson, B., Youngstrom, E. A., Hinshaw, S., Bass, J. M., ... y Salloum, I. (2012). Creativity and bipolar disorder: Touched by fire or burning with questions? Clinical Psychology $\quad$ Review, $\quad 32(1), \quad$ 1-12. https://doi.org/10.1016/j.cpr.2011.10.001

Kéri, S. (2009). Genes for psychosis and creativity: a promoter polymorphism of the neuregulin 1 gene is related to creativity in people with high intellectual achievement. Psychological Science, 20(9), 1070-1073. https://doi.org/10.1111/j.1467-9280.2009.02398.x

Kleibeuker, S. W., Stevenson, C. E., Van der Aar, L., Overgaauw, S., van Duijvenvoorde, A. C. y Crone, E. A. (2017). Training in the adolescent brain: An fMRI training study on divergent thinking. Developmental Psychology, 53(2), 353-365. https://doi.org/10.1037/dev0000239

Klimenko, O. (2008). La creatividad como un desafío para la educación del siglo XXI. Educación y Educadores, 11(2) 191-210.

Kohli, A. y Kaur, M. (2006). Wisconsin card sorting test: Normative data and experience. Indian Journal of Psychiatry, 48(3), 181-184. https://doi.org/10.4103/0019-5545.31582

Liu, S., Erkkinen, M. G., Healey, M. L., Xu, Y., Swett, K. E., Chow, H. M. y Braun, A. R. (2015). Brain activity and connectivity during poetry composition: Toward a multidimensional model of the creative process. Human Brain Mapping, 36(9), 3351-3372. https://doi.org/10.1002/hbm.22849

Luft, C. B., Zioga, I., Banissy, M. J. y Bhattacharya, J. (2017). Relaxing learned constraints through cathodal TDCS on the left dorsolateral prefrontal cortex. Scientific Reports, 7(1), 2916-2917. https://doi.org/10.1038/s41598-017-03022-2 
Murillo, F. J. y Hernández-Castilla, R. (2014). Liderando escuelas justas para la justicia social. Revista Internacional de Educación para la Justicia Social (RIEJS), 3(2), 13-32.

Myers, D. y Twenge. J. M. (2012). Social psychology. Nueva York, NY: McGraw-Hill.

Neddens, J., Vullhorst, D., Paredes, D. y Buonanno, A. (2009). Neuregulin links dopaminergic and glutamatergic neurotransmission to control hippocampal synaptic plasticity. Communicative E Integrative Biology, 2(3), 261-264. https://doi.org/10.4161/cib.2.3.7825

O'Shea, D. y Fieo, R. (2015). Individual differences in fluid intelligence predicts inattentional blindness in a sample of older adults: A preliminary study. Psychological Research, 79(4), 570578. https://doi.org/10.1007/s00426-014-0594-O

Penagos-Corzo, J. C. (2014). Creativity as an attitude: An approach to origins of creativity. En F. Reisman (Ed.), Creativity in business (pp. 162-172). Philadelphia, PA: KIE Books Series.

Penagos-Corzo, J. C. (2017). Variables psicobiológicas asociadas a la creatividad. Hallazgos y desafío. Entorno UDLAP, 3, 15-23. https://doi.org/10.11144/Javeriana.upsy 16-2.ppwp

Penagos-Corzo, J. C., Olvera, M. y Pintado, S. (2017). Propiedades psicométricas del work preference inventory (WPI) en una muestra de adultos jóvenes mexicanos. Universitas Psychologica 16(2), 5-25.

Pidgeon, L. M., Grealy, M., Duffy, A. B., Hay, L., McTeague, C., Vuletic, T., ... y Gilbert, S. J. (2016). Functional neuroimaging of visual creativity: A systematic review and metaanalysis. Brain E Behavior, 6(10), 41-57. https://doi.org/10.1002/brb3.540

Poblano, A. (1991). Las neurociencias y la filosofía. Salud Pública de México, 33(1), 88-93.

Prakash, R. y Zhongde, D. (2013). More wide-spread approach needed to explore visual creativity. Annals of Indian Academy of Neurology, 16(1), 128-129. https://doi.org/10.4103/09722327.107665

Reuter, M., Roth, S., Holve, K. y Hennig, J. (2006). Identification of first candidate genes for creativity: A pilot study. Brain Research, 1069(1), 190-197. https://doi.org/10.1016/j.brainres.2005.11.046

Reynolds, M. H. (2008). Hope in adults, ages 20-59, with advanced stage cancer. Palliative \& Supportive Care, 6(3), 259-264. https://doi.org/10.1017/S1478951508000394

Rhodes M. (1961). An analysis of creativity. En S. G. Isaksen (Ed.), Frontiers of creativity research: Beyond the basics (pp. 216-222). Buffalo, NY: Bearly.

Rowe, G., Hirsh, J. B. y Anderson, A. K. (2007). Positive affect increases the breadth of attentional selection. Proceedings of the National Academy of Sciences, 104(1), 383-388. https://doi.org/10.1073/pnas.0605198104

Runco, M. A. (2004). Creativity. Annual Review of Psychology, 55(1), 657-687.

Runco, M. A. (2007). Creativity. Theories and themes: Research, development, and practice. San Diego, CA: Elsevier Academic Press. https://doi.org/10.1 146/annurev.psych.55.090902.141502

Runco, M. A. y Jaeger, G. J. (2012). The standard definition of creativity. Creativity Research Journal, 24(1), 92-96. https://doi.org/10.1080/10400419.2012.650092

Runco, M. A., Noble, E. P., Reiter-Palmon, R., Acar, S., Ritchie, T. y Yurkovich, J. M. (2011). The genetic basis of creativity and ideational fluency. Creativity Research Journal, 23(4), 376-380. https://doi.org/10.1080/10400419.2011.621859

Sawyer, K. (2011). The cognitive neuroscience of creativity: A critical review. Creativity Research Journal, 23(2), 137-154. https://doi.org/10.1080/10400419.2011.571191 
Skvortsova, V., Degos, B., Welter, M., Vidailhet, M. y Pessiglione, M. (2017). A selective role for dopamine in learning to maximize reward but not to minimize effort: Evidence from patients with Parkinson's disease. The Journal of Neuroscience, 37(25), 6087-6097. https://doi.org/10.1523/JNEUROSCI.2081-16.2017

Sprugnoli, G., Rossi, S., Emmendorfer, A., Rossi, A., Liew, S., Tatti, E., ... y Santarnecchi, E. (2017). Neural correlates of eureka moment. Intelligence, 62, 99-118. https://doi.org/10.1016/j.intell.2017.03.004

Sternberg, R. (1986). A triangular theory of love. Psychological Review, 93(2), 119-135. https://doi.org/10.1037/0033-295X.93.2.119

Swartwood, M. O., Swartwood, J. N. y Farrell, J. (2003). Stimulant treatment of ADHD: Effects on creativity and flexibility in problem solving. Creativity Research Journal, 15(4), 417-419. https://doi.org/10.1207/S15326934CRJ1504_9

Volf, N. V., Kulikov, A. V., Bortsov, C. U. y Popova, N. K. (2009). Association of verbal and figural creative achievement with polymorphism in the human serotonin transporter gene. Neuroscience Letters, 463(2), 154-157. https://doi.org/10.1016/j.neulet.2009.07.070

Vrieze, E., Ceccarini, J., Pizzagalli, D. A., Bormans, G., Vandenbulcke, M., Demyttenaere, K., ... y Claes, S. (2013). Measuring extrastriatal dopamine release during a reward learning task. Human Brain Mapping, 34(3), 575-586.

Wacker, J. (2017). Effects of positive emotion, extraversion, and dopamine on cognitive stabilityflexibility and frontal EEG asymmetry. Psychophysiology, 27, 11-36.

Young, S. N. (2007). How to increase serotonin in the human brain without drugs. Journal of Psychiatry $\mathbb{\Xi}^{2}$ Neuroscience, 32(6), 394-399.

Zhang, S. y Zhang, J. (2017). The association of TPH genes with creative potential. Psychology of Aesthetics, Creativity, and the Arts, 11(1), 2-9. https://doi.org/10.1037/aca0000073

\section{Breve CV del autor}

\section{Julio C. Penagos-Corzo}

Doctor en Ciencias del Lenguaje, con estudios de Doctorado en Investigación Psicológica. Maestría en Calidad de la Educación y Licenciatura en Psicología con especialidad en Psicología Clínica para Adultos, entre otros estudios. Dentro de las asocicaciones a las que está afiliado o es miembro, destacan la American Psychological Association, la Society for Neuroscience y la International Society for Comparative Psychology. Ha sido coordinador del Grupo de Trabajo de Psicología Experimental y Comparada de la Interamerican Society of Psychology (SIP) (2015-2017). Actualmente es vicepresidente para la región México, Centroamérica y el Caribe de dicha Sociedad (2017-2019). Dentro de su producción científica puede destacarse la invención (patentada) del dispositivo de observación "Caja de Escape-Exploración-Vitación en Campo Restringido". ORCID ID: https://orcid.org/o000-0002-4879-3033. Email: julioc.penagos@udlap.mx 